\title{
BMJ Open What is the value of fibre-endoscopic evaluation of swallowing (FEES) in neurological patients? A cross-sectional hospital-based registry study
}

\author{
Tobias Braun, ${ }^{1}$ Martin Juenemann, ${ }^{1}$ Maxime Viard, ${ }^{1}$ Marco Meyer, ${ }^{1}$ Sven Fuest, \\ Iris Reuter, ${ }^{1}$ Manfred Kaps, ${ }^{1}$ Mario Prosiegel, ${ }^{2}$ Christian Tanislav ${ }^{1}$
}

To cite: Braun T, Juenemann M, Viard M, et al. What is the value of fibre-endoscopic evaluation of swallowing (FEES) in neurological patients? A cross-sectional hospital-based registry study. BMJ Open 2018;8:e019016. doi:10.1136/ bmjopen-2017-019016

- Prepublication history and additional material for this paper are available online. To view these files, please visit the journal online (http://dx.doi org/10.1136/bmjopen-2017019016).

Received 9 August 2017 Revised 16 December 2017 Accepted 5 February 2018
Check for updates

${ }^{1}$ Department of Neurology, University Hospital Giessen and Marburg, Giessen, Germany ${ }^{2}$ Institute of German Linguistics, Ludwig-Maximilians-University, Giessen, Germany

Correspondence to Dr Tobias Braun; tobias.braun@neuro.med.unigiessen.de

\section{ABSTRACT}

Objectives Fibre-endoscopic evaluation of swallowing (FEES) to detect dysphagia is gaining more and more importance as a diagnostic tool. Therefore, we have investigated the impact of FEES in neurological patients in a clinical setting.

Design Cross-sectional hospital-based registry.

Setting Primary acute care in a neurological department of a German university hospital.

Participants 241patients with various neurological diseases who underwent FEES procedure.

Primary and secondary outcome measures Dysphagia and related comorbidities.

Results 267 FEES were performed in 241 patients with various neurological diagnoses. Dysphagia was diagnosed in $68.9 \%$ of the patients. In only $33.1 \%$ of the patients, appropriate oral diet was chosen prior to FEES. A relevant dysphagia occurred more often in patients with structural brain lesions $(83.1 \%$ vs $65.3 \%, P=0.001$ ), patients with dysphagia had a longer hospitalisation (median 18 (IQR 12-30) vs 15 days (IQR 9.75-22.75), $\mathrm{P}=0.005$ ) and had a higher mortality $(8.4 \%$ vs $1.3 \%, P=0.041)$. When the oral diet was changed, we observed a lower pneumonia rate (36\% vs $50 \%, P=0.051)$ and a lower mortality ( $3.7 \%$ vs $11.3 \%, P=0.043$ ) in comparison to no change of oral diet. A restriction of oral diet was identified more often in older patients (median 75 years (IQR 66.3-82 years) vs median 72 years (IQR $60-79$ years), $\mathrm{P}=0.01$ ) and in patients with structural brain lesions $(86.8 \%$ vs $73.1 \%$ $\mathrm{P}=0.05)$.

Conclusion On clinical investigation, dysphagia was misjudged for the majority of the patients. FEES might help to compensate this drawback, revising the diet regime in nearly $70 \%$ of the patients.

\section{BACKGROUND}

Dysphagia is a common complication in neurological diseases with aspiration pneumonia as a leading cause of death in stroke, multiple sclerosis, amyotrophic lateral sclerosis, Parkinson's disease or dementia. ${ }^{1-5} \mathrm{In}$ elderly patients suffering from infections, a concomitant aspiration pneumonia results

\section{Strengths and limitations of this study}

- Performance of fibre-endoscopic evaluation of swallowing (FEES) by experienced examiners in a standardised manner.

- Considering the current literature, our study has included the highest number $(n=241)$ of neurological patients systematically examined with FEES.

- Single-centre study, which might lead to a centrespecific bias in diagnostic and therapeutic measures, influencing data acquisition.

in increased morbidity and mortality. ${ }^{6} 7$ Dysphagia determines therefore the immediate prognosis of ill patients, and due to the functional link to the central and peripheral nerve system, it is of particular relevance to neurological patients. ${ }^{7}$

Apart from a physical examination performed by physicians or speech and language therapists, diagnostic tools have been developed to investigate the swallowing function. ${ }^{8}$ Two procedures, the videofluoroscopic evaluation of swallowing and the fibre-endoscopic evaluation of swallowing (FEES) have entered the clinical practice for this purpose. The latter works without radiation exposure and it can be easily repeated; it can be performed at bedside and even in uncooperative or unconscious patients. FEES is therefore gaining more and more importance in the examination of neurological patients. ${ }^{9}{ }^{10}$ However, systematic studies providing the overall benefit of this procedure in neurological patients are currently missing.

Therefore, the aim of the presented study is to assess the value of FEES for unselected neurological patients regarding the benefit of judging the swallowing function and the related short-term outcome. 


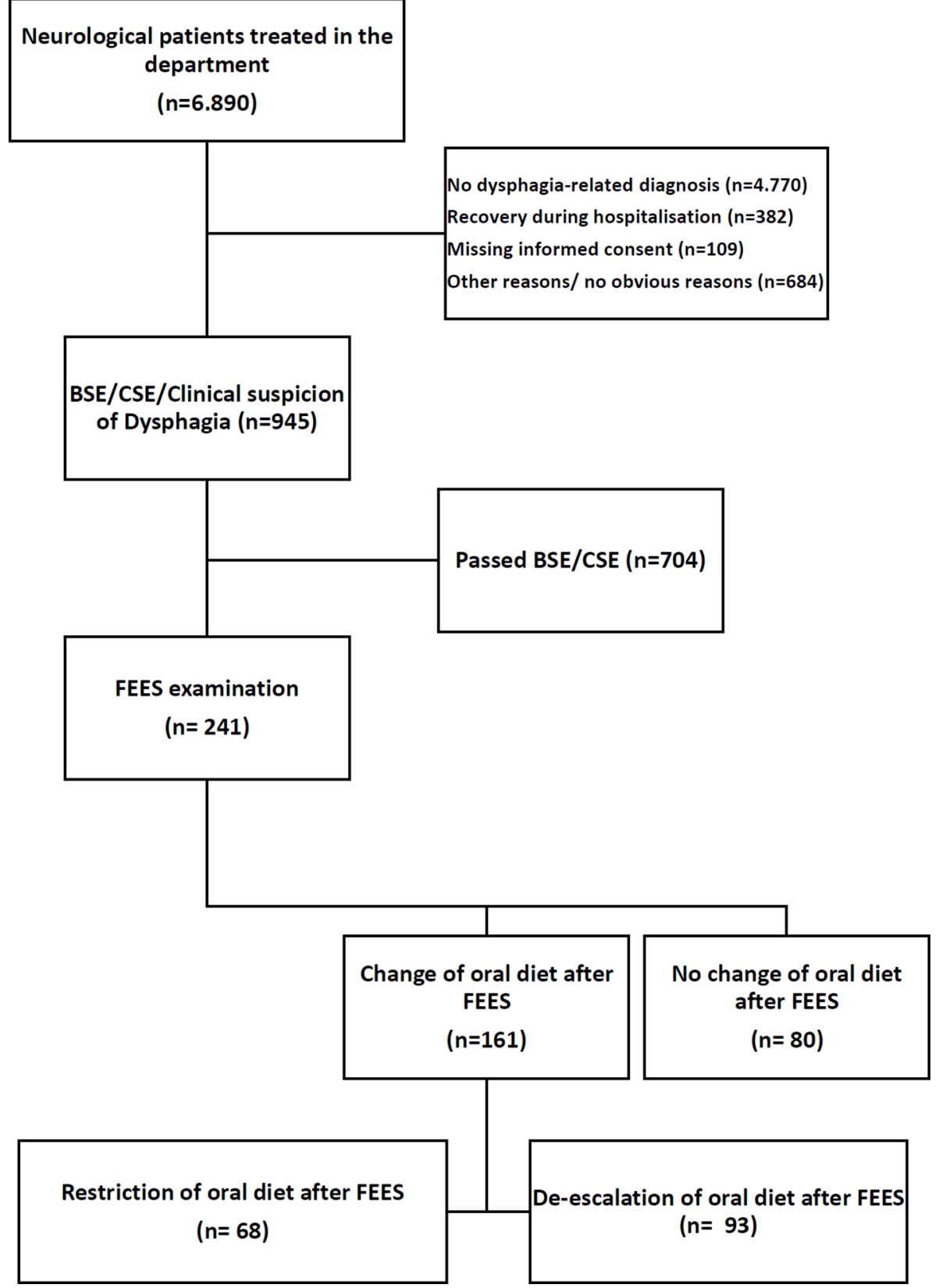

Figure 1 Strobe diagram of screening process and decisions after FEES. BSE, bedside screening examination; CSE, comprehensive swallowing examination; FEES, fibre-endoscopic evaluation of swallowing.

\section{METHODS}

FEES has been performed in patients who had a stroke with pathological bedside screening examination (BSE). In patients with other aetiologies, it has been performed when there were pathological findings in the comprehensive swallowing examination (CSE) conducted by a speech and language therapist (SLT). An indication for CSE was a clinical suspicion of dysphagia, that is, in patients with newly diagnosed motor neuron disease or in those showing clinical signs of dysphagia (eg, wet voice and/or coughing when drinking, etc). In our department, we use the Gugging Swallowing Screen (GUSS) as a BSE for stroke-associated dysphagia. The GUSS consists of four subtests. In the first subtest, vigilance, the ability to cough and swallowing of saliva are assessed. The next three steps evaluate the patient's ability to safely swallow semisolid, liquid and solid food. In each subtest, a maximum of five points can be reached. The level of points determines the patient's severity of dysphagia. Due to the degree of severity, different diet recommendations are given. ${ }^{11}$ The screening process is depicted in figure 1.

The oral diet prior to FEES was chosen by the attending physician and an SLT based on the findings in the CSE. In patients who had a stroke, oral diet was chosen according to the instructions of the GUSS. For quality control reasons, findings gathered in examinations were documented systematically. All FEES procedures were performed in a standardised manner by experienced physicians (see below). 


\section{Diagnoses}

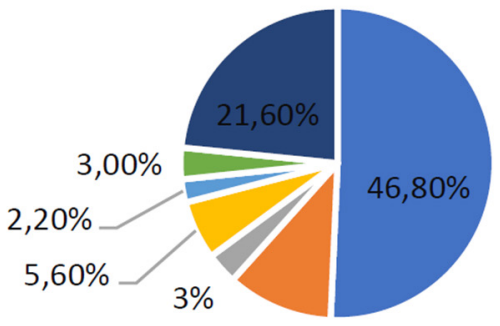

$10,10 \%$

$$
\begin{aligned}
& \text { - Ischemic stroke } n=125 \quad \text { - Intracranial bleeding } n=27 \\
& \text { - Traumatic brain injury } n=8 \text { - Movement disorders } n=15 \\
& \text { - Motor neuron disease } n=6 \text { - Intracranial tumor } n=8
\end{aligned}
$$

Figure 2 Disease entities detected in patients in percentage and absolute.

\section{Patients}

All patients treated in our department from January 2014 to September 2016 who underwent FEES were considered for the analysis. The data documented in the database included: age, sex, length of stay in hospital, diagnosis, presence of brain lesions (such as a new or old ischaemic stroke, an intracerebral bleeding, a tumour, a cerebral atrophy, etc), occurrence of pneumonia (defined as a clinical diagnosis of pneumonia; determined by the treating physician), treatment in the intensive care unit, mortality, presence of dysphagia and type of oral intake (before and after FEES). To acquire data and to use them for scientific analyses, an ethical approval was obtained from the local ethical committee (Justus-Liebig University, Giessen; protocol number 208/16).

\section{FEES}

FEES is a videoendoscopic nasolaryngeal swallowing study. We performed FEES following the standardised FEES protocol according to Langmore ${ }^{12}$ : a small endoscope (about $4 \mathrm{~mm}$ in diameter) was introduced through the inferior nasal meatus and the epipharynx in the mesopharynx. Swallowing of saliva and different consistencies of food and liquids, penetration, aspiration, localisation and amount of residues as well as patients' reactions (such as coughing) were visualised and documented. By definition, penetration is entering of any material into the airway (above the level of the vocal folds), aspiration means entering of any material below the level of the vocal folds. In the first step of the procedure, anatomical changes, management of saliva and movements of swallowing related structures were assessed. Then, we tested pudding-thick consistency (thickened water), normal water and solid food. All consistencies were applied three times. If one of the consistencies appeared unsafe, we left out the corresponding consistence. Based on the findings in FEES, the appropriate oral diet was chosen for the patients. All FEES procedures were performed or supervised by an experienced investigator.

\section{Outcome measurements}

Oral intake and the degree of dysphagia severity were measured by using the Functional Oral Intake Scale (FOIS) and the Fiberoptic Endoscopic Dysphagia Severity Scale (FEDSS), respectively.

FOIS is a seven-tiered scale ranging from $1=$ no oral intake at all $(\mathrm{NPO}=$ nil per os) to $7=$ fulloral intake without restrictions (see online supplementary appendix 1). ${ }^{13}$ The data of the functional oral intake scale were categorised in either NPO (FOIS $=1$ ), partial oral intake $($ FOIS=2-6) or full oral intake (FOIS=7). De-escalation of the oral diet was defined as a positive change on the FOIS, whereas restriction of the oral diet was defined as a negative change.

To define the overall severity of dysphagia, we used the FEDSS developed by Dziewas and coworkers. ${ }^{14}$ The FEDSS is a six-tiered scale originally designed to use for patients who had a stroke (see online supplementary appendix 2). All parameters are recorded in a standardised way.

To evaluate the value of performing FEES in neurological patients, the following parameters were correlated with baseline data and dependent factors:

- Dysphagia as defined by a FEDSS score of $\geqq 2$.

- Oral intake status as calculated by the FOIS and its overall change and type of change after FEES.

\section{Statistical analyses}

Absolute and relative frequencies were calculated based on cross-tables. For comparing relative frequencies, a two-tailed Fisher's exact test was used. Continuous variables were analysed by calculating the median value and the IQR (25\% percentile and $75 \%$ percentile). Non-parametric data were analysed employing the Mann-Whitney U test. All statistical analyses were performed with the SPSS V.22.0 (IBM Company).

\section{RESULTS}

\section{Patients' characteristics}

Two hundred and sixty-seven FEES were performed in 241 patients. In 23 patients $(9.5 \%)$, the procedure was repeated at least once. Among those patients, an improvement of dysphagia was noted in 12 cases $(52.1 \%)$. The subsequent examination revealed an increased severity of dysphagia in one patient and in a second patient, previously diagnosed with no dysphagia, aspiration was detected during a repeated examination.

One hundred and forty patients were male (52.4\%), the median age was 73 years (IQR $61-80$ years). One hundred and nine patients $(45.2 \%)$ were treated on the intensive care unit. In $46.8 \%$ of the patients, an ischaemic stroke was diagnosed. The different disease entities detected in our patients are summarised in figure 2. The group classified as 'other' consisted of patients with 
Table 1 Baseline characteristics of neurological patients and differences in patients with and without dysphagia

\begin{tabular}{lcccc}
\hline & $\begin{array}{l}\text { Total } \\
\text { cohort (n=241) }\end{array}$ & $\begin{array}{l}\text { Normal swallowing } \\
\text { function (n=75) }\end{array}$ & $\begin{array}{l}\text { Relevant } \\
\text { dysphagia (n=166) }\end{array}$ & P \\
\hline Sex (number of male patients) & $140(58.1 \%)$ & $41(54.6 \%)$ & $99(59.6 \%)$ & 0.401 \\
\hline Age (median, IQR) & $73(61-80)$ & $71.5(59-79.5)$ & $73(62-81)$ & 0.261 \\
\hline Ischaemic stroke & $125(51.9 \%)$ & $34(45.3 \%)$ & $91(57.8 \%)$ & 0.165 \\
\hline Intensive care unit & $109(45.2 \%)$ & $34(45.3 \%)$ & $75(45.2 \%)$ & $>0.999$ \\
\hline Brain lesion & $187(77.6 \%)$ & $49(65.3 \%)$ & $138(83.1 \%)$ & 0.001 \\
\hline Pneumonia & $98(40.7 \%)$ & $28(37.3 \%)$ & $70(42.2 \%)$ & 0.481 \\
\hline Length of stay in hospital in days (median, IQR) & $17(11-29)$ & $15(9.75-22.75)$ & $18(12-30)$ & 0.005 \\
\hline Death & $15(6.2 \%)$ & $1(1.3 \%)$ & $14(8.4 \%)$ & 0.041 \\
Change in oral diet & $176(65.9 \%)$ & $61(75.3 \%)$ & $115(61.8 \%)$ & 0.36 \\
\hline Restriction & $70(26.2 \%)$ & $2(2.5 \%)$ & $68(36.6 \%)$ & $<0.001$ \\
\hline NPO started & $47(17.6 \%)$ & $0(0 \%)$ & $47(25.3 \%)$ & $<0.001$ \\
\hline De-escalation & $106(39.7 \%)$ & $59(72.8 \%)$ & $47(25.3 \%)$ & $<$ \\
\hline PEG on admission & $7(2.9 \%)$ & $3(4 \%)$ & $4(2.4 \%)$ & 0.682 \\
\hline PEG procedure in hospital & $49(20.3 \%)$ & $14(18.7 \%)$ & $35(21.1 \%)$ & 0.731 \\
\hline PEG at discharge & $54(22.4 \%)$ & $17(22.7 \%)$ & $37(22.3 \%)$ & $>0.999$ \\
\hline
\end{tabular}

NPO, nil per os (no oral intake); PEG, percutaneous endoscopic gastrostomy tube.

heterogeneous diagnoses (epileptic seizures, dementia, Guillain-Barré syndrome, degenerative changes of the cervical spine, etc).

One hundred and ninety-four patients $(80.5 \%)$ had CT imaging of the brain, $69(28.6 \%)$ underwent MRI. Forty-eight patients $(19.9 \%)$ had both a CT and MRI scan, whereas 22 (9.1) patients had no imaging at all. One hundred and eighty-seven patients had a brain lesion detected in CT scan or MRI (8 tumours, 125 new ischaemic lesions, 27 bleedings, 27 other lesions (old ischaemic lesions, unspecific white matter lesions, cortical atrophy, etc)). Ninety-eight patients (40.7\%) developed pneumonia, 15 patients died during hospitalisation $(6.2 \%)$. Initially, 140 patients $(52.4 \%)$ had NPO, 58 patients $(24.1 \%)$ had partial oral intake and 43 patients $(16.1 \%)$ had full oral intake. One hundred and eight patients $(44.8 \%)$ were dependent on a nasogastric feeding tube prior to FEES and seven patients $(2.9 \%)$ on a percutaneous endoscopic gastrostomy tube. Patients' characteristics are presented in table 1 .

No side effects, that is, laryngospasm, syncope or non-self-limiting epistaxis occurred, two patients $(0.8 \%)$ had mild epistaxis after FEES.

\section{FEES examination}

The median overall FEDSS score of the entire study population was 4 (IQR 1-6). FEES revealed no sign of dysphagia (FEDSS $=1)$ for 75 patients $(31.1 \%)$, whereas dysphagia (FEDSS 2-6) was diagnosed in 166 persons $(68.9 \%)$.

An oral diet was more often de-escalated in patients without dysphagia $(72.8 \%$ vs $25.3 \%, \mathrm{P}<0.0001)$ and was more often restricted in patients with dysphagia patients with a normal swallowing function $(2.5 \%$ vs $36.6 \%$,
$\mathrm{P}<0.001)$. As for 26 patients $(10.8 \%)$ with a full oral intake, FEES showed a critical dysphagia and as a result, the diet was re-evaluated. Out of these 26 patients, 16 $(61.5 \%)$ had a partial oral intake and $38.5 \%$ patients had NPO after FEES. Changes in oral diet after FEES can be seen in figure 1.

Patients with brain lesions were more often diagnosed with dysphagia $(65.3 \%$ vs $83.1 \%, \mathrm{P}=0.001)$. Patients with dysphagia stayed longer in hospital (median 15 (IQR 9.75-22.75) vs 18 (IQR12-30) days, $\mathrm{P}=0.005$ ) and had a higher mortality $(8.4 \%$ vs $1.3 \%, \mathrm{P}=0.041)$. Results are summarised in table 1.

\section{Differences between patients with a change in the oral diet}

A total of 161 patients $(66.8 \%)$ had a change in the oral diet, 93 of them $(57.8 \%)$ were de-escalated and $(42.2 \%)$ a restriction was necessary in 68 patients. NPO was recommended after the examination in 47 of those 68 patients restricted in the oral diet $(69.1 \%)$. Patients without a change of the oral diet had a higher rate of pneumonia ( $40.4 \%$ vs $36 \%, \mathrm{P}=0.051)$ and a higher mortality as compared with those with a change in the oral diet $(10 \%$ vs $3.7 \%, \mathrm{P}=0.043)$. Results are summarised in table 2.

\section{Differences between patients with de-escalation and restriction of the oral diet}

In the patients with a change in oral diet, a restriction of oral diet was indicated more often in older patients (median 75 (IQR 66.25-82) years old vs 72 (IQR 60-79), $\mathrm{P}=0.01)$, in patients who had an ischaemic stroke $(64.7 \%$ vs $46.2 \%, \mathrm{P}=0.025$ ) or patients with any other brain lesion ( $86.8 \%$ vs $73.1 \%, \mathrm{P}=0.05$ ) as compared with de-escalation of oral diet. There was also a higher mortality in patients with restriction in oral diet as compared with de-escalation 
Table 2 Baseline characteristics of neurological patients and differences of patients with and without a change of the oral diet

\begin{tabular}{lcccc}
\hline & $\begin{array}{l}\text { Total } \\
\text { cohort }(\mathbf{n = 2 4 1})\end{array}$ & $\begin{array}{l}\text { No change in oral } \\
\text { diet }(\mathbf{n = 8 0})\end{array}$ & $\begin{array}{l}\text { Change in oral } \\
\text { diet }(\mathbf{n = 1 6 1 )}\end{array}$ & $\mathbf{P}$ \\
\hline Sex (number of male patients) & $140(58.1 \%)$ & $45(56.3 \%)$ & $95(59 \%)$ & 0.782 \\
\hline Age (median, IQR) & $73(61-80)$ & $74.5(60.25-80.75)$ & $72(61-80)$ & 0.286 \\
\hline Ischaemic stroke & $125(51.9 \%)$ & $38(47.5 \%)$ & $87(54 \%)$ & 0.412 \\
\hline Intensive care unit & $109(45.2 \%)$ & $43(53.8 \%)$ & $66(41 \%)$ & 0.074 \\
\hline Brain lesion & $187(77.6 \%)$ & $60(75 \%)$ & $127(78.9)$ & 0.515 \\
\hline Pneumonia & $98(40.7 \%)$ & $40(50 \%)$ & $58(36 \%)$ & 0.051 \\
\hline Length of stay in hospital in days (median, IQR) & $17(11-29)$ & $17.5(12-33)$ & $17(11-26)$ & 0.242 \\
\hline Death & $15(6.2 \%)$ & $9(11.3 \%)$ & $6(3.7 \%)$ & 0.043 \\
\hline PEG on admission & $7(2.9 \%)$ & $5(6.3 \%)$ & $2(1.2 \%)$ & 0.042 \\
\hline PEG procedure in hospital & $49(20.3 \%)$ & $17(21.3 \%)$ & $32(19.9 \%)$ & 0.865 \\
\hline PEG at discharge & $54(22.4 \%)$ & $20(25 \%)$ & $34(21.1 \%)$ & 0.515 \\
\hline
\end{tabular}

PEG, percutaneous endoscopic gastrostomy tube.

$(7.4 \%$ vs $1.1 \%, \mathrm{P}=0.082)$. Results are summarised in table 3 .

\section{DISCUSSION}

FEES showed relevant dysphagia in $166(68.9 \%)$ of 241 unselected neurological patients. After performing the FEES, the diet was revised in $66.8 \%$ of the patients. A restriction of oral intake was indicated for predominantly elderly patients and those suffering from stroke or those with other structural brain lesions. Relevant dysphagia was associated with a higher mortality and a longer duration of hospitalisation.

Different studies identified dysphagia as a strong factor associated with the bad outcome in many disorders. ${ }^{1-5} 15-17$ Therefore, establishing the right diagnosis with initiation of the appropriate therapeutic measures is of major relevance. In this context, FEES seems to be a promising tool of identifying patients at risk. With this procedure, a considerable number of patients with relevant dysphagia resulting in the immediate adjustment of the oral diet could be identified. In line with investigations in other populations, patients diagnosed with dysphagia in our study had a longer period of hospitalisation and a higher risk for poor outcome. It can be expected that pneumonia is the main complication associated of a poor outcome; however, our analysis showed no significant differences in pneumonia rates between patients with compared with patients without dysphagia. Thus, some other factors might determine the development of dysphagia in neurological patients. As demonstrated in our study, dysphagia was associated with the presence of structural brain lesions, which could be attributed to the complexity of the swallowing process. Swallowing is controlled and regulated by complex supramedullary networks, so brain lesions causing a relevant swallowing dysfunction seem to be an appropriate finding. ${ }^{18} 19$

Table 3 Differences of patients with de-escalation or restriction of the oral diet

\begin{tabular}{|c|c|c|c|c|}
\hline & $\begin{array}{l}\text { Change in oral } \\
\text { diet }(n=161)\end{array}$ & $\begin{array}{l}\text { De-escalation of } \\
\text { oral diet }(n=93)\end{array}$ & $\begin{array}{l}\text { Restriction of oral } \\
\text { diet }(n=68)\end{array}$ & $\mathbf{P}$ \\
\hline Sex (number of male patients) & $95(59 \%)$ & $55(59.1 \%)$ & $40(58.8 \%)$ & $>0.999$ \\
\hline Age (median, IQR) & $72(61-80)$ & $72(60-79)$ & 75 (66.25-82) & 0.01 \\
\hline Ischaemic stroke & $87(54 \%)$ & $43(46.2 \%)$ & $44(64.7 \%)$ & 0.025 \\
\hline Intensive care unit & $66(41 \%)$ & $49(52.7 \%)$ & $17(25 \%)$ & 0.001 \\
\hline Brain lesion & $127(78.9)$ & $68(73.1 \%)$ & $59(86.8 \%)$ & 0.05 \\
\hline Pneumonia & $58(36 \%)$ & $38(40.9 \%)$ & $20(29.4 \%)$ & 0.183 \\
\hline Length of stay in hospital in days (median, IQR) & $17(11-26)$ & $17(11.75-27.25)$ & $18(11-31)$ & 0.95 \\
\hline Death & $6(3.7 \%)$ & $1(1.1 \%)$ & $5(7.4 \%)$ & 0.082 \\
\hline PEG on admission & $2(1.2 \%)$ & $2(2.2 \%)$ & 0 & \\
\hline PEG procedure in hospital & $32(19.9 \%)$ & $19(20.4 \%)$ & $13(19.1 \%)$ & $>0.999$ \\
\hline PEG at discharge & 34 (21.1\%) & $21(22.6 \%)$ & 13 (19.1\%) & 0.697 \\
\hline
\end{tabular}

PEG, percutaneous endoscopic gastrostomy tube. 
The initial diet regime was maintained after performance of FEES for only $33.1 \%$ of the investigated patients. Despite extensive clinical expertise, the established diagnosis regarding the swallowing function was incorrect in nearly every second patient; $10.8 \%$ of the patients had full oral intake although they would have needed a restriction. A lack of awareness of dysphagia, the inability of clinical examinations and screening tests to detect silent aspirations or methodological reasons might also explain this result. ${ }^{20}$ Therefore, our results underline the necessity of performing elaborate dysphagia diagnostics on a routine basis and they support recent trends implementing FEES examination as a standard procedure in severely affected neurological patients.

A restriction of an oral diet was indicated more often in older patients. The age-related impairment of physiological function, also called 'presbyphagia' might be responsible for this observation. ${ }^{21}$ Since the vast majority of neurological patients in general are of an advanced age, presbyphagia needs to be taken into consideration when interpreting FEES findings. A structural brain lesion in addition to pre-existing presbyphagia might explain the distinct severity of dysphagia in our study group. Mortality and pneumonia rate were higher in patients that had no change of their oral diet. This might sound surprising at first, but this group included, apart from non-dysphagic patients, patients that were on a restricted diet or NPO based on the results of the BSE, CSE or clinical judgement. The group of NPO patients had severe dysphagia and a group of them aspirated saliva, which might explain the higher rate of pneumonia and mortality. Those complications might have been prevented by intubation or tracheotomy, but for most patients, this was not an option for the patient or the treating physician. Again, this demonstrates the lack of awareness for dysphagia.

A selection bias considering a large number of intensive care patients must be taken into account when interpreting our results. Since those patients are more severely affected, that is, by stroke, our findings could have overestimated the number of neurological patients affected by dysphagia, which might also explain the high frequency of pneumonia as compared with other researchers. ${ }^{22}$ Because of ethical reasons, no control group (without FEES) was set up: the risk of pneumonia and pneumonia-related death was considered too high. These are the main limitations of the study. However, the study design represents the clinical routine with a preselection of patients by using a BSE or CSE followed by instrumented diagnostics. So far only one study by Bax and coworkers has been published about the effect of FEES on the outcome in 220 neurological patients. ${ }^{23}$ However, in this study only some patients underwent FEES. Whereas in our study, all 241 patients underwent FEES procedure.

\section{CONCLUSIONS}

By implementing FEES, we could detect signs of dysphagia in $68.9 \%$ of our neurological patients. Dysphagia was associated with the presence of a structural brain lesions, a higher mortality and a longer duration of hospitalisation. A change of the oral diet was associated with a lower incidence of pneumonia and a lower mortality. Due to our findings, only $33.1 \%$ of the patients had an adequate oral diet. As most screening tests for dysphagia do not cover non-stroke patients and cannot detect silent aspiration, using FEES at a low threshold for all neurological patients might help identifying patients at risk with this safe and fast bedside assessment tool. It ensures safety when deciding on the type of the oral intake and brings the benefit of a marked reduction in mortality and morbidity.

Contributors TB, MJ, MK and CT: Conceptualisation. TB, MV, MM and IR: FEES examinations. TB, MJ and MP: Analysis of data and statistics. TB and CT: Preparation of original draft. All authors: Review and editing; ICMJE criteria for authorship read and agree with manuscript results and conclusions.

Funding This research received no specific grant from any funding agency in the public, commercial or not-for-profit sectors.

Competing interests None declared.

Patient consent Not required.

Ethics approval For the data acquisition and their use for scientific analyses, an ethical approval was obtained from the local ethical committee (Justus-Liebig University, protocol number 208/16).

Provenance and peer review Not commissioned; externally peer reviewed.

Data sharing statement The authors declare that the data supporting the findings of this study are available within the article. The data that support the findings of this study are not publically available due to local medical data protection policies.

Open Access This is an Open Access article distributed in accordance with the Creative Commons Attribution Non Commercial (CC BY-NC 4.0) license, which permits others to distribute, remix, adapt, build upon this work non-commercially, and license their derivative works on different terms, provided the original work is properly cited and the use is non-commercial. See: http://creativecommons.org/ licenses/by-nc/4.0/

(c) Article author(s) (or their employer(s) unless otherwise stated in the text of the article) 2018. All rights reserved. No commercial use is permitted unless otherwise expressly granted.

\section{REFERENCES}

1. Heuschmann PU, Kolominsky-Rabas PL, Misselwitz B, et al. Predictors of in-hospital mortality and attributable risks of death after ischemic stroke: the German Stroke Registers Study Group. Arch Intern Med 2004;164:1761-8.

2. Chen A, Garrett CG. Otolaryngologic presentations of amyotrophic lateral sclerosis. Otolaryngol Head Neck Surg 2005;132:500-4.

3. Cabre M, Serra-Prat M, Palomera E, et al. Prevalence and prognostic implications of dysphagia in elderly patients with pneumonia. Age Ageing 2010;39:39-45.

4. Mamolar Andrés S, Santamarina Rabanal ML, Granda Membiela $\mathrm{CM}$, et al. Swallowing disorders in Parkinson's disease. Acta Otorrinolaringol Esp 2017;68:15-22.

5. Prosiegel M, Schelling A, Wagner-Sonntag E. Dysphagia and multiple sclerosis. Int MS J 2004;11:22-31.

6. LaCroix AZ, Lipson S, Miles TP, et al. Prospective study of pneumonia hospitalizations and mortality of U.S. older people: the role of chronic conditions, health behaviors, and nutritional status. Public Health Rep 1989;104:350-60.

7. Warnecke T, Ritter MA, Kroger B, et al. Fiberoptic endoscopic dysphagia severity scale predicts outcome after acute stroke. Cerebrovasc Dis 2009;28:283-9.

8. Wieseke A, Bantz D, Siktberg L, et al. Assessment and early diagnosis of dysphagia. Geriatr Nurs 2008;29:376-83.

9. Frieling T. The role of the endoscopist in the stroke unit. Visc Med 2016;32:53-7. 
10. Smithard DG. Dysphagia management and stroke units. Curr Phys Med Rehabil Rep 2016;4:287-94.

11. Trapl M, Enderle P, Nowotny $M$, et al. Dysphagia bedside screening for acute-stroke patients: the Gugging Swallowing Screen. Stroke 2007;38:2948-52.

12. Langmore SE. Endoscopic evaluation and treatment of swallowing disorders. New York, NY: Thieme, 2001.

13. Crary MA, Mann GD, Groher ME. Initial psychometric assessment of a functional oral intake scale for dysphagia in stroke patients. Arch Phys Med Rehabil 2005;86:1516-20.

14. Dziewas R, Warnecke T, Olenberg S, et al. Towards a basic endoscopic assessment of swallowing in acute stroke-development and evaluation of a simple dysphagia score. Cerebrovasc Dis 2008;26:41-7.

15. Arnold M, Liesirova K, Broeg-Morvay A, et al. Dysphagia in acute stroke: incidence, burden and impact on clinical outcome. PLoS One 2016;11:e0148424.

16. Bray BD, Smith CJ, Cloud GC, et al. The association between delays in screening for and assessing dysphagia after acute stroke, and the risk of stroke-associated pneumonia. J Neurol Neurosurg Psychiatry 2017;88:25-30.
17. Scmidt EV, Smirnov VE, Ryabova VS. Results of the seven-year prospective study of stroke patients. Stroke 1988;19:942-9.

18. Hamdy S, Aziz Q, Rothwell JC, et al. The cortical topography of human swallowing musculature in health and disease. Nat Med 1996;2:1217-24.

19. Dziewas R, Sörös $P$, Ishii R, et al. Neuroimaging evidence for cortical involvement in the preparation and in the act of swallowing. Neuroimage 2003;20:135-44

20. Bours GJ, Speyer R, Lemmens J, et al. Bedside screening tests vs. videofluoroscopy or fibreoptic endoscopic evaluation of swallowing to detect dysphagia in patients with neurological disorders: systematic review. J Adv Nurs 2009;65:477-93.

21. Muhle P, Wirth R, Glahn J, et al. [Age-related changes in swallowing. Physiology and pathophysiology]. Nervenarzt 2015;86:440-51.

22. Hannawi $\mathrm{Y}$, Hannawi B, Rao CP, Cpv R, et al. Stroke-associated pneumonia: major advances and obstacles. Cerebrovasc Dis 2013;35:430-43.

23. Bax L, McFarlane M, Green E, et al. Speech-language pathologistled fiberoptic endoscopic evaluation of swallowing: functional outcomes for patients after stroke. J Stroke Cerebrovasc Dis 2014;23:e195-e200. 